\title{
Factors associated with anxiety in critically ill patients: A prospective observational cohort study
}

\section{Authors:}

Maria I Castillo, RN, $\mathrm{PhD}^{\mathrm{a}, \mathrm{b}, \mathrm{c}, \text { \& e }}$, Marie $\underline{\text { Cooke, }} \mathrm{RN}, \mathrm{PhD}^{\mathrm{a}, \mathrm{b}}$, Bonnie Macfarlane, $\mathrm{MPH}^{\mathrm{a}, \mathrm{b}}$, Leanne M $\underline{\text { Aitken, RN, PhD, FACN, FAAN, FACCCN }}$ a, b, c \& d

aschool of Nursing and Midwifery, Griffith University, Nathan Campus, 170 Kessels Road, Nathan, Queensland 4111, Australia.

${ }^{b}$ NHMRC Centre of Research Excellence in Nursing (NCREN), Menzies Health Institute Queensland, Griffith University, Nathan Campus, 170 Kessels Road, Nathan, Queensland 4111, Australia.

'Intensive Care Unit, Princess Alexandra Hospital, Ipswich Road, Woolloongabba, Queensland 4102, Australia.

${ }^{\mathrm{d}}$ School of Health Sciences, City University London, Northampton Square London EC1V 0HB, United Kingdom.

Professor Leanne Aitken: l.aitken@griffith.edu.au

Professor Marie Cooke: m.cooke@griffith.edu.au

Bonnie Macfarlane: bonnie.macfarlane@griffith.edu.au

\section{${ }^{\text {e}}$ Corresponding author:}

Dr. Maria Castillo, Nursing Practice Development Unit, Princess Alexandra Hospital, Ipswich Road, Woolloongabba, Queensland 4102, Australia.

phone: 0731767256

fax: +61 732407356 
email: isabel.castillo@griffithuni.edu.au

\section{Institution where work was conducted:}

Griffith University and Princess Alexandra Hospital Intensive Care Unit, Brisbane, Queensland, Australia.

\section{Ethical approval:}

The Griffith University (NRS/35/12/HREC) and Princess Alexandra Hospital Ethics Committees (HREC/12/QPAH/173) approved this research.

\section{Financial support:}

Intensive Care Foundation Research Grant and Australian College of Critical Care Nurses Research Grant.

\section{Conflict of interest: none.}

\section{Authors' contributions}

MIC made a substantial contribution to the design of the study, enrolment of patients, collection of data, data analyses, interpretation of data and wrote the manuscript. LA and MC were responsible for the design of the study and made a substantial contribution to the interpretation of data and writing of the manuscript. BM conducted the mixed model analysis and assisted with data interpretation and writing of the manuscript. MIC, LA, MC and BM revised the manuscript critically for important intellectual content. All authors read and approved the final manuscript. 


\begin{abstract}
Background: Anxiety is an unpleasant emotion that most intensive care patients experience. This emotion is an important issue in intensive care settings because of its prevalence, adverse effects and severity. Little is known about the factors associated with state and trait anxiety during critical illness.
\end{abstract}

Objectives: To describe the patterns of state anxiety reported by intensive care patients, and identify factors associated with state and trait anxiety.

Design: Prospective observational cohort study.

Settings: One mixed intensive care unit in Brisbane, Australia.

Participants: Adults ( $\mathrm{n}=141, \geq 18$ years), admitted to the intensive care unit for $\geq 24$ hours; able to communicate verbally or non-verbally; understand English; and, open their eyes spontaneously or in response to voice.

Methods: Outcomes were state anxiety as measured by the Faces Anxiety Scale and trait anxiety as measured by the State-Trait Anxiety Inventory. Pre-intensive care factors tested for possible associations with both state and trait anxiety were: age, gender, marital status, employment status, level of education, smoking status, personality trait of optimism and evidence of mental health care/treatment. Intra-intensive care factors tested were: reason for admission to the intensive care unit, delirium, pain, airway status, hours of mechanical ventilation, severity of illness, days of stay in intensive care, exposure to corticosteroids, opioids, benzodiazepines, anxiolytics, antidepressants, betablockers, anesthetic agents and analgesics; length of sedation and analgesia and total doses of sedatives and analgesics. 
Results: Of 141 participants, 98 (70\%) were male with an average age of 54 (Standard Deviation [SD]: \pm 15 ) years and stayed in intensive care for about 4 (Interquartile Range [IQR]: 3-7) days. The majority ( $\mathrm{n=115}$; 82\%) of participants experienced state anxiety at least once during their stay in intensive care, with 57\% reporting moderate to severe levels. Moderate levels of anxiety were predominantly reported from days 6 to 12 and then decreased. Trait anxiety in these participants (median 36 IQR: 29-47) was very similar to the Australian population. Factors related to state anxiety in intensive care were pain and trait anxiety. Factors associated with trait anxiety were trait optimism, state anxiety, evidence of mental health care/treatment and age.

Conclusion: This study provides a better understanding of contributing factors for anxiety in the critically ill. Trait anxiety and state anxiety were significantly associated with each other, namely, patients who were anxious by nature experienced higher levels of state anxiety, which persisted throughout their stay in the intensive care unit. Recognising the importance of state and trait anxiety assessments using validated tools and determining ways to manage anxiety in the critically ill are critical aspects of the intensive care nurses role.

\section{Keywords}

Critically ill, intensive care unit, risk factors, state anxiety, trait anxiety. 


\section{Introduction}

Anxiety is an unpleasant emotion that most intensive care unit patients experience (Tate et al., 2012). This emotion comprises two components: state and trait. State anxiety alerts the individual of imminent danger enabling them to prepare and deal with the threat (Doenges et al., 2010). It helps the body to respond to stressful situations through physiological arousal and feelings of tension, apprehension, nervousness and worry. Trait anxiety is a more stable characteristic of an individual's personality. It is the tendency of a person to become state anxious (Spielberger, 1983, Spielberger, 1966, Spielberger and Reheiser, 2009).

Although state anxiety is a normal adaptive mechanism in human beings, it can become detrimental if it impairs an individual's ability to function (Steimer, 2002). State anxiety can progress to agitation or panic and even modify physiological function (e.g. promote the formation of gastric ulcers or dysrhythmias); becoming pathological and representing a clinical concern (Doenges et al., 2010, Moser and Dracup, 1996, Steimer, 2002). High levels of anxiety, particularly when sustained, are less adaptive and might contribute to the development of adverse emotional outcomes after the intensive care experience (Nelson et al., 2000).

A high prevalence of state anxiety has been identified in critically ill patients, especially in those requiring mechanical ventilation. In a descriptive study including 192 ventilated patients state anxiety was found in all of them, with low levels in $20 \%$ $(n=38)$, moderate to severe levels in $62 \%(n=119)$ and high levels in $18 \%(n=35)$ of the patients (Chlan, 2003). In addition, a significantly higher prevalence of anxiety was reported in mechanically ventilated patients than in non-ventilated ( $74.2 \%$ versus $25.8 \%$ respectively, $\mathrm{p}=0.02$ ) in seriously ill patients at risk of dying (Puntillo et al., 2010). In a 
qualitative investigation exploring this emotion in 30 intensive care patients, all the patients exhibited anxiety at some stage during the study (Tate et al., 2011).

A high proportion of intensive care patients usually report moderate to high levels of anxiety despite receiving sedation and/or analgesia. In 106 Australian patients (89\% ventilated) severe anxiety was reported by 35\% of patients who received sedation $(n=45)$ and $66 \%$ of patients who did not ( $n=61)$ (McKinley et al., 2004). Several years later, using similar measurement instruments, McKinley and Madronio (2008) reported lower levels of anxiety in 100 non-ventilated patients. However, more than a quarter (28\%) of patients still reported moderate to severe anxiety, and half of them (14\%) had received sedation (McKinley and Madronio, 2008).

The need for intensive care treatment is accompanied by several physical, psychological and environmental sources of distress. Physical sources of distress such as invasive mechanical ventilation, may lead to physiologically and psychologically distressing experiences since the patients are not able to verbalise their feelings, symptoms or wants. In fact, spells of terror, nervousness when left alone, and sleeping disturbances have been associated with endotracheal intubation (Rotondi et al., 2002). Psychological sources of distress such as confusion, fear, panic, and frustration are common while receiving intensive care (Tate et al., 2011). Examples of environmental sources of distress are noise, lights and lack of privacy (Puntillo et al., 2010, Yava et al., 2011). Despite the sources of distress during intensive care treatment being documented in the literature, there is little empirical research exploring sources of distress as potential risk factors for anxiety in the intensive care unit. Statistical approaches such as multivariate analysis to determine unique contributions and rule out the influence of confounding factors are needed. 
Although there is some description of anxiety in the critically ill population, the majority of the evidence available provides information only about the state component. Recent reports have shown a relationship between trait anxiety and symptoms of anxiety, depression and posttraumatic stress disorder in intensive care survivors (Castillo et al., 2015a, Castillo et al., 2015b). These findings are in line with previous reports in other populations such as survivors of rectal cancer (Ristvedt and Trinkaus, 2009). As research in the field of psychology suggests that trait anxiety can be modified using tailored interventions, it seems beneficial to also explore trait anxiety in the intensive care patient (Clark et al., 2003, Jackson et al., 2012, Tang et al., 2009). Since trait anxiety has been associated with the development of adverse emotional outcomes, the investigation of anxiety in the critically ill patient is imperative.

In this current research, we aimed to describe the patterns of state anxiety reported by patients throughout their stay in the intensive care unit, and test a number of variables as risk factors for anxiety (state and trait) during critical illness. Factors were categorized into two groups: pre-intensive care factors and intra-intensive care factors. Pre-intensive care factors tested for possible associations with both state and trait anxiety were: age, gender, marital status, employment status, level of education, smoking status, personality trait of optimism and evidence of mental health care/treatment. Intra-intensive care factors tested were: reason for admission to the intensive care unit, delirium, pain, airway status, hours of mechanical ventilation, severity of illness, days of stay in the intensive care unit, exposure to corticosteroids, opioids, benzodiazepines, anxiolytics, antidepressants, beta-blockers, anesthetic agents and analgesics; length of sedation and analgesia and total doses of sedatives and analgesics. State anxiety and trait anxiety were considered as both outcome variables 
and risk factor of each other. Other factors such as memories of endotracheal intubation, memories of anxiety, fear, panic as well as environmental factors where out of the scope of this research. 


\section{Methods}

Participants in this analysis were enrolled in a prospective follow-up study that investigated anxiety, depression and posttraumatic stress symptoms in intensive care survivors over six months after discharge from the intensive care unit (Castillo et al., 2013, Castillo et al., 2015a, Castillo et al., 2015b). Participants ( $n=141)$ were from one closed mixed medical/surgical/trauma intensive care unit of a tertiary metropolitan public hospital located in Brisbane, Australia. This 25-bed intensive care unit provides 24-hour intensivist led care, with a registered nurse/patient ratio of 1:1. Data were collected prospectively between September 2012 and February 2013. The study protocol has previously been published (Castillo et al., 2013), but a summary of the methods is provided below. The current study is a preplanned sub-study analysis of this research.

Inclusion criteria were adult patients ( $\geq 18$ years), admitted to the intensive care unit for $\geq 24$ hours; able to communicate verbally or non-verbally; understand English; and, open their eyes spontaneously or in response to voice. As patients woke up and became interactive at different times, they were not recruited on a particular day of their stay in the intensive care unit. Patients were invited to participate in the project as soon as meeting the inclusion criteria and their assent (verbal or non-verbal) was sought prior to data collection in the intensive care unit. This was followed by written consent being sought once the patients were in the hospital wards, and able to provide informed consent. The relevant Human Research Ethics Committees approved this research.

Pre-intensive care factors collected in the hospital wards using a set of questionnaires included: marital status, employment status and level of education, preintensive care medications (benzodiazepines, anxiolytics, antidepressants, corticoids, 
opioids, and beta-blockers), smoking status, personality trait of optimism (Life Orientation Test-Revised), personality trait of anxiety (Trait component of the SateTrait Anxiety Inventory Form Y-2) and evidence of mental health care/treatment (Scheier et al., 1994, Spielberger, 1983). Participants who answered "Yes” to either of the following two question was considered to have positive evidence of mental health care/treatment prior to the intensive care admission: (1) Have you ever visited a general practitioner or a mental health professional for symptoms of psychological distress or emotional problems? (2) Were you taking benzodiazepines, anxiolytics or antidepressants medications within the 12 months prior to the intensive care admission? Information about previous mental health history, pre-intensive care medications (corticoids, opioids and beta-blockers) and personality trait of optimism were collected because the literature suggests a possible association between these factors and adverse emotional outcomes after the intensive care experience (Bryant et al., 2009, Fletcher et al., 2010, Myhren et al., 2009, Schelling et al., 2001). Age and gender were collected from electronic patient notes held in the intensive care unit.

Intra-intensive care factors collected from electronic patient notes held in the intensive care unit included: type of admission (medical, surgical, trauma, cardiac surgery), delirium (Confusion Assessment Method - Intensive Care Unit), hours of mechanical ventilation (invasive and non-invasive), airway status (tracheostomy, endotracheal tube, natural), Acute Physiology And Chronic Health Evaluation III score, length of intensive care unit stay (days), length of hospital stay (days) and pain using the Critical-Care Pain Observation Tool (Cook et al., 2002, Ely et al., 2001, Gelinas et al., 2006, Gelinas et al., 2009). Data on drugs administered included exposure to corticosteroids, opioids, benzodiazepines, anxiolytics, antidepressants, beta-blockers, 
anesthetic agents and analgesics; length of sedation and analgesia (hours of propofol, midazolam, morphine, fentanyl, ketamine, oxycodone infusion); and total doses of sedatives and analgesics (propofol, midazolam, morphine, fentanyl, ketamine, oxycodone and paracetamol).

The primary outcomes of this study were both components of anxiety (state and trait) in the critically ill patient. State anxiety and trait anxiety were also considered as a risk factor of each other. Levels of state anxiety were self-reported twice daily in the intensive care unit (morning 8-11 am and evening 4-7 pm) using the Faces Anxiety Scale (McKinley et al., 2004). These timeframes were selected because we wanted to identify any difference between morning assessments (usually busier intensive care unit environment) and evening assessments (usually quieter intensive care unit environment). We selected the Face Anxiety Scale because it is a practical tool, specially designed to measure state anxiety in intensive care settings. The Faces Anxiety Scale consists of a scale with five faces with each face representing a different level of anxiety. The score ranges from 1 (no anxiety) to 5 (extreme anxiety). The criterion validity of this scale was $0.64(\mathrm{p}<0.001)$ in mechanically ventilated patients (Pearson's correlation coefficient between the self-report of anxiety on the Faces Anxiety Scale and clinical judgment of patient's anxiety) (McKinley et al., 2004).

Before approaching for the assessment of anxiety, the following information was recorded: airway status (tracheostomy, endotracheal tube, natural), mechanical ventilation status (invasive, non-invasive, non-ventilation), delirium status (Confusion Assessment Method- Intensive Care Unit), oxygen saturation, pain score and sedation (total dose of sedatives and analgesics as well as total hours of continuous infusion of sedoanalgesia). Then, the participants were shown the Faces Anxiety Scale and asked to 
choose the face that better represented how much anxiety they felt at the moment of assessment. The answer could be provided by a verbal or a nonverbal response; i.e. they could point to the relevant face.

Trait anxiety was assessed using the Trait component of the State-Trait Anxiety Inventory for adults Form Y-2 (Spielberger, 1983). This assessment was performed when participants were in the hospital wards and sufficiently awake to be able to answer questions in this inventory. The State-Trait Anxiety Inventory is a self-report 20-items measure based on a 4-point Likert scale with scoring going from 20-80 with higher scores indicating greater levels of trait anxiety. The State-Trait Anxiety Inventory is a well-validated and recognised tool for the assessment of trait anxiety and has previously been used with survivors of critical illness at a similar time point in their recovery process (Jones et al., 2003, Kress et al., 2003). Trait anxiety was assessed in the wards and not in the intensive care unit for two reasons: completion of the State-Trait Anxiety Inventory requires a patient able to maintain attention for about 10 minutes: and, trait personalities are stable patterns of cognition, affect and behavior that are relatively consistent across time and situations (American Psychiatric Association, 2013). Thus, trait anxiety would have been unlikely to change in such a short period (between intensive care unit stay and assessment in the wards) and without a specific intervention to modify this personality trait. The principal investigator and the intensive care unit Research Nurse conducted the state anxiety assessments in the intensive care unit and assisted the participants (when needed due to physical impairment) with the surveys in hospital wards.

We performed power analysis a priori using $\mathrm{G}^{*}$ Power to determine the sample size needed for this study (Faul et al., 2009). Multiple regression test (fixed model, $\mathrm{R}^{2}$ 
increase) with a power of $80 \%$, a significance level of alpha $=0.05$ and a medium size effect (0.15) were used. In addition, we expected a maximum of seven variables to be included in the final model and a mortality rate of $10 \%$. Thus, the estimated sample size for this study was 104 participants.

The outcome variable "state anxiety” was derived from repeated measures taken during the participants' intensive care unit stay (twice a day). In order to incorporate state anxiety into the multivariable modelling, it was essential to determine a single score that represented the entire intensive care unit stay. We extensively explored the first state anxiety measure in the intensive care unit and several aggregate variables (e.g. mean state anxiety and median state anxiety). However, we selected the mean value because it was the strongest at accounting for correlations amongst observations in the same cluster with an Interclass Correlation Coefficient of 0.53.

Distributions of continuous variables were assessed with frequency histograms and tests for normality. Relationships between each risk factor and the outcome variables (trait and state anxiety) were tested using appropriate inferential statistics (continuous, binary and nominal/ordinal risk factors used Spearman's correlation, Mann-Whitney U test, Kruskal-Wallis, respectively). Variables associated at P-value ( $p$ ) $\leq 0.2$ were considered in subsequent multivariable analyses.

Multiple linear regression was used to identify factors significantly associated with state anxiety. Model building used forward selection where variables significant at the bivariate level $(p \leq 0.2)$ were added into the model one at a time, repeating this process until none improved the model. Variables not significant at the bivariate level but identified in the literature as important were tested in the final model. The same process was repeated to determine factors significantly associated with trait anxiety. 
Regression diagnostics were performed informally (graphical) and formally (statistical) to verify the analysis met the assumptions underlying multiple linear regression. Diagnostic tests included assessing the normality and homoscedasticity of residuals, degree of multicollinearity amongst risk factors, the linearity assumption between the outcome variable and risk factors and identification of outliers. Statistical analyses were conducted using Stata version 13 (StataCorp, College Station, Texas) and SPSS version 21 (IBMCorp, 2013, StataCorp, 2013).

\section{Results}

Of 797 patients screened during the enrollment period 600 were excluded: 597 stayed in the intensive care unit for less than 24 hours, and three were younger than 18 years old. One hundred and ninety-seven patients agreed to participate in this study and reported on their levels of state anxiety during intensive care treatment. One hundred and twenty patients completed the State-Trait Anxiety Inventory in the wards (Figure 1). There were no significant differences in baseline demographics (age, gender, Acute Physiology And Chronic Health Evaluation III score, length of intensive care unit stay and hospital stay) or state anxiety between those who assented to intensive care data collection and those who completed the State-Trait Anxiety Inventory.

Participants were predominantly male (70\%) and relatively young (mean $54.1 \pm 15.3$ years). The majority were in a relationship (61\%) and worked (57\%). Evidence of mental health care/treatment prior to the intensive care admission was reported by $37 \%$ of participants and just under a third (28\%) smoked. Approximately half of the participants were admitted with medical diagnoses (49\%), followed by surgical (21\%), trauma (17\%) and cardiac surgery (13\%). Most participants received mechanical ventilation (82\%) for about 52 (Interquartile Range [IQR]: 13-148) hours, 
and sedation and analgesia with propofol ( $n=119,84 \%)$, fentanyl ( $n=111,79 \%)$, midazolam ( $n=49,35 \%)$ and morphine $(n=37,26 \%)$. Participants stayed in the intensive care unit for about four days and fifteen days in hospital.

Eighty-two percent $(n=115)$ of participants reported state anxiety at least once during their stay in intensive care, with $57 \%(n=80)$ reporting moderate to severe levels. Because most of the participants had reduced consciousness during the first hours in the intensive care unit, they were able to report state anxiety status only half of their intensive care days. Participants reported moderate to severe state anxiety on $44 \%$ of these days. While the levels of state anxiety fluctuated over time, there was no significant difference between morning and afternoon assessments (2.4 Standard Deviation $[\mathrm{SD}] \pm 1.0$ versus $2.3 \mathrm{SD} \pm 1.0$, $\mathrm{t}(88)=0.28, p=0.779)$. Moderate levels of anxiety were predominantly reported from days 6 to 12 and then decreased (Figure 2). The trait component of anxiety in these participants (median 36 [IQR: 29-47]) was similar to the Australian population (Crawford et al., 2011).

Factors associated with state anxiety on univariate analysis $(p \leq 0.2)$ included: trait anxiety $\left(\mathrm{r}_{\mathrm{s}}=0.32, p<0.001\right)$, evidence of mental health care/treatment $\left(\mathrm{r}_{\mathrm{s}}=-0.22\right.$, $\mathrm{p}=0.01)$, trait optimism $\left(\mathrm{r}_{\mathrm{s}}=-0.15, \mathrm{p}=0.1\right)$ and pain $(\mathrm{rho}=0.16, p=0.06)$. After multivariable model adjustment both trait anxiety and pain remained significant. Model results indicated trait anxiety as the largest contributor to state anxiety (beta $=0.29$ ) followed by pain (beta=0.18) (Table 1$)$.

Thirteen factors were significantly associated $(p \leq 0.20)$ with trait anxiety on univariate analysis: age, evidence of mental health care/treatment, trait optimism, state anxiety, smoking, length of hospital stay, length of propofol infusion, total dose of propofol, total dose of midazolam, total dose of morphine, length of fentanyl infusion, 
total dose of oxycodone and pain (Table 2). Several factors remained significantly associated with trait anxiety after adjustment including age, trait optimism, state anxiety, and evidence of mental health care/treatment (Table 3).

\section{Discussion}

This observational study showed that intensive care patients with mixed diagnoses suffer emotional distress during their intensive care admission. Despite our participants having similar trait anxiety levels to the Australian norms, the majority of participants experienced moderate to severe levels of state anxiety during intensive care treatment. The prevalence and severity of state anxiety found in our sample are comparable to the prevalence and severity reported in other studies (Chlan and Savik, 2011, Chlan, 2004, McKinley et al., 2004).

Our participants were able to report state anxiety status on half of their intensive care days because they were unconscious or too ill to be assessed the other half. Of the days of assessment, moderate to severe state anxiety was reported by almost half of the participants. Self-reported levels of state anxiety demonstrated minimal fluctuation from days 6 to 12, with average scores showing moderate levels over this period (Figure 2). During these days in the intensive care unit, participants might have been capable of perceiving stressors such as pain, noise and endotracheal tube and, therefore, their levels of state anxiety were higher. After day 12, participants may have become accustomed to the intensive care environment, staff and routine, resulting in a decrease in their anxiety levels. As participants in this study reported state anxiety after 24 hours of being in the intensive care unit, levels for the initial 24 hours are unknown. In addition, as this 
component was only assessed during participants' intensive care unit stay, state anxiety levels after the intensive care discharge, but still in hospital wards, are also unknown. Despite these limitations, the information provided about state anxiety in this study is important because this is only the second study to describe patients' self-report of state anxiety throughout the intensive care stay.

Trait anxiety and state anxiety were significantly associated with each other. Participants who were anxious by nature experienced higher levels of state anxiety. This finding is consistent with the theory behind the anxiety concept developed by Spielberger (Spielberger, 1983, Spielberger, 1966, Spielberger and Reheiser, 2009). Given trait anxiety levels in our sample were similar to the ones found in the general Australian population, it is reasonable to suggest that anyone with high anxiety personality trait admitted to the intensive care unit is at greater risk of experiencing state anxiety during intensive care treatment. Measuring trait anxiety prior to admission to the intensive care unit in elective patients and as soon as possible after admission in emergency patients might help clinicians to identify patients at risk of experiencing high levels of state anxiety during intensive care treatment. During patients’ intensive care stay, state anxiety should be assessed systematically, and clinicians should use this assessment to guide treatment in the intensive care unit.

Participants with lower levels of pain reported significantly less state anxiety. Because pain may be perceived as a very distressing symptom, it is not surprising that these two unpleasant symptoms were associated with each other in our participants (Puntillo et al., 2010). Effective strategies for pain management are fundamental to alleviate both pain and state anxiety in the critically ill (Puntillo et al., 2010). Trait anxiety and evidence of mental health care/treatment prior to the intensive care 
admission were also significantly associated. The relationship between trait anxiety and general mental health in the intensive care population requires additional exploration.

Lower levels of trait anxiety were observed in older participants, suggesting a protective effect of ageing on anxiety outcomes. To our knowledge, this relationship has not previously been reported in the intensive care patient. However, there is evidence that age-related reduced prefrontal-amygdala structural connectivity is associated with lower levels of trait anxiety in healthy adults (Clewett et al., 2014).

Optimistic participants reported significantly lower levels of trait anxiety. The relationship between these two trait personalities has previously been reported in healthy participants and patients hospitalised for chronic diseases (Kepka et al., 2013, Raikkonen et al., 1999). No study reporting this association in the intensive care patient was located. This finding is important because trait optimism was identified as a predictor of less anxiety and depression symptoms after one year in intensive care survivors (Myhren et al., 2010). Trait anxiety was also associated with anxiety, depression and posttraumatic stress symptoms over six months after discharge from the intensive care unit (Castillo et al., 2015a, Castillo et al., 2015b). In addition, the interaction between these two trait personalities in the development of adverse emotional outcomes in the intensive care survivor is yet to be tested. Thus, age, trait optimism and evidence of mental health care/treatment prior to the intensive care admission were all factors significantly associated with trait anxiety, which in turn together with pain were associated with state anxiety in the intensive care unit (Table 1 and 3).

Although a number of risk factors were examined and tested, no other demographic (age, gender, marital status, etc.) or clinical (sedatives, analgesics, 
mechanical ventilation, etc.) variables were significantly associated with state anxiety in the intensive care unit. Given that sedation is often administered to treat agitation and anxiety (Barr et al., 2013), it was anticipated that we would find a significant statistical relationship between sedation and anxiety, but this was not the case. Discrepancies between patients' self-reported anxiety and clinician's observations of anxiety might explain this lack of association (O'Brien et al., 2001). The management of anxiety in intensive care is often based on clinicians' detection of it, which is usually done through observation of physiological and behavioural manifestations (Tate et al., 2012). Unfortunately, clinicians' observations of physiological and behavioural manifestations of anxiety are limited since delirium and pain share similar physiological and behavioural characteristics with anxiety. These similarities may lead to erroneous symptom interpretation. It might be the case that the association between state anxiety and sedation was not found in this sample because sedation was not administered to treat anxiety.

These findings further underline the importance of the assessment of both components of anxiety (state and trait) in the critically ill patient. State anxiety can be easily assessed in the intensive care unit by the use of validated self-reported measures such as the Faces Anxiety Scale or the Visual Analog Scale-Anxiety (Chlan, 2004, McKinley et al., 2004). Trait anxiety could be an assessment performed when patients are able to respond to the trait component of the State-Trait Anxiety Inventory either by the bedside intensive care nurse or intensive care outreach nurses (who follow-up patients in the wards). The feasibility of trait anxiety assessment needs to be tested. Early assessment of state and trait anxiety in intensive care patients is vital to put in place simple non-pharmacological interventions to alleviate anxiety in the intensive care 
unit, promote comfort and potentially reduce the risk of adverse outcomes during recovery. Music therapy, education, stress management, reassurance, encouragement or coaching and psychological interventions such as cognitive behavioural therapy are examples of non-pharmacological interventions that could be provided by intensive care nurses to reduce anxiety in intensive care patients and its potential detrimental consequences (Chlan et al., 2013, Moser et al., 2003, Peris et al., 2011, Tate et al., 2011). However, more research is needed in this area so that the effectiveness of these interventions in the intensive care context as well as their long-term effects in intensive care survivors can be established.

Our study was limited to adult patients who were $\geq 24$ hours in a general intensive care unit and therefore only generalisable to patients who spend at least 24 hours in the intensive care unit. Although a sub-study, it was planned at the time of conception of the larger study (Castillo et al., 2013). While we assessed the levels of state anxiety, we did not explore the reasons why patients were anxious such as environmental factors. It might have been beneficial to collect this information in a qualitative or retrospective manner although there are some previous studies examining state anxiety in the intensive care unit that are available to aid interpretation of these data (Rotondi et al., 2002, Tate et al., 2012).

\section{Conclusion}

This study provides a better understanding of contributing factors for anxiety in the critically ill. Trait anxiety and state anxiety were significantly associated with each other, namely, patients who were anxious by nature experienced higher levels of state 
anxiety, which persisted throughout the intensive care stay. It also demonstrates that it is crucial for intensive care nurses to recognise the importance of state and trait anxiety assessments using validated tools and determine ways to manage anxiety in the critically ill. Non-pharmacological strategies such as music therapy or psychological interventions have been shown to be effective in reducing anxiety in the intensive care patient, however, more research is needed to test the long-term effectiveness of these interventions in intensive care survivors.

\section{Acknowledgements}

The Intensive Care Foundation and the Australian College of Critical Care Nurses Novice Researcher Grants funded this study. We thank Dr. Robert Ware for advising on statistical analysis. We also thank the intensive care unit Research Nurses Lena James and Kelly Perkins for their assistance with data collection. 
What is already known about the topic?

- Critically ill patients often experience anxiety during ICU stay

- Anxiety during critical illness has recently been associated with the development of anxiety, depression and posttraumatic stress symptoms after the ICU experience.

What this paper adds

- State anxiety persists throughout the ICU stay, with highest levels reported from day 6 to 12

- Factors associated with state anxiety during critical illness include trait anxiety and pain

- Factors associated with trait anxiety during critical illness include age, trait optimism, state anxiety, and evidence of mental health treatment

- Early assessment and management of anxiety during ICU treatment might help to reduce the risk of adverse emotional outcomes after critical illness. 


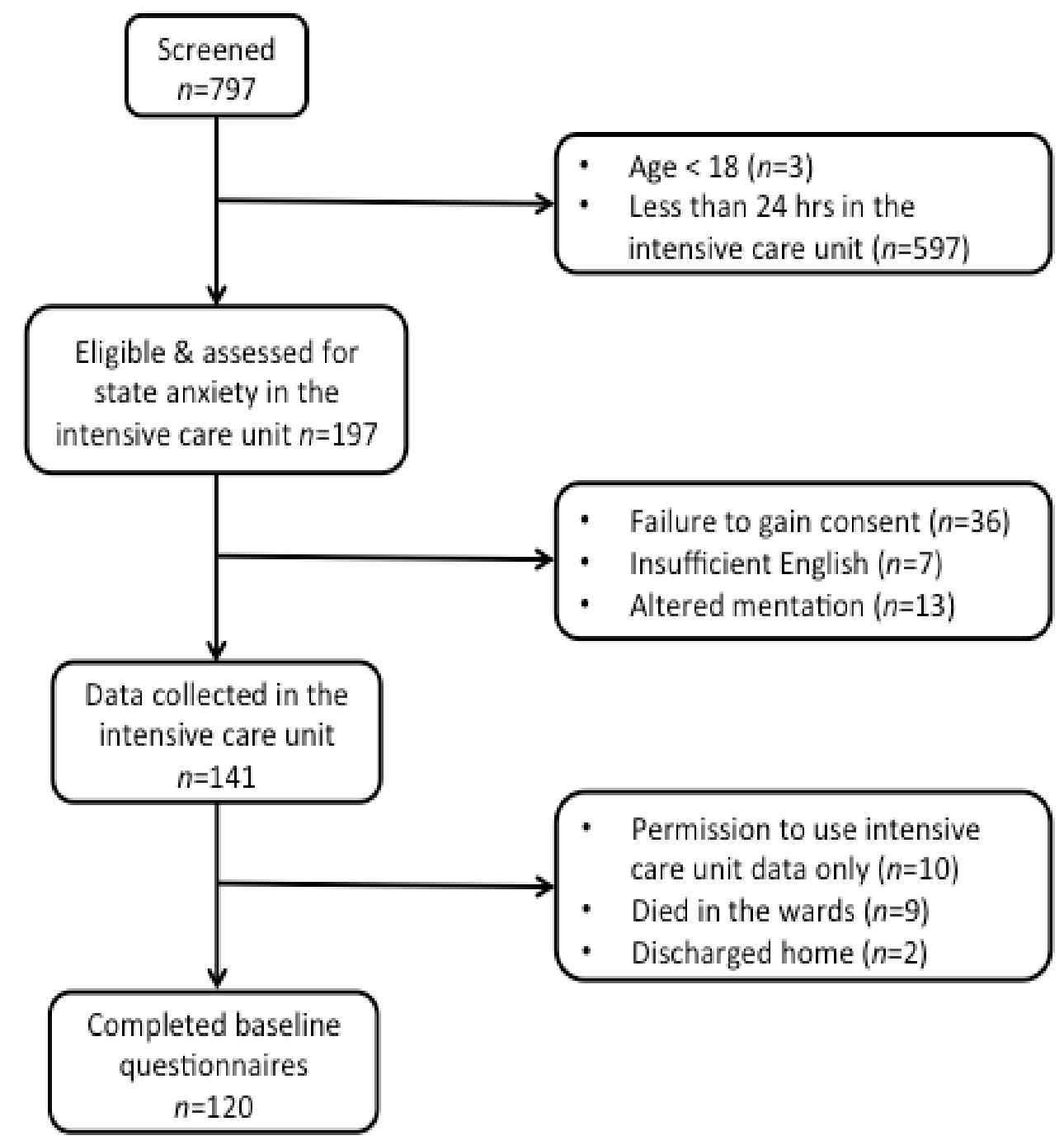

Figure 1. Participant flow through study 


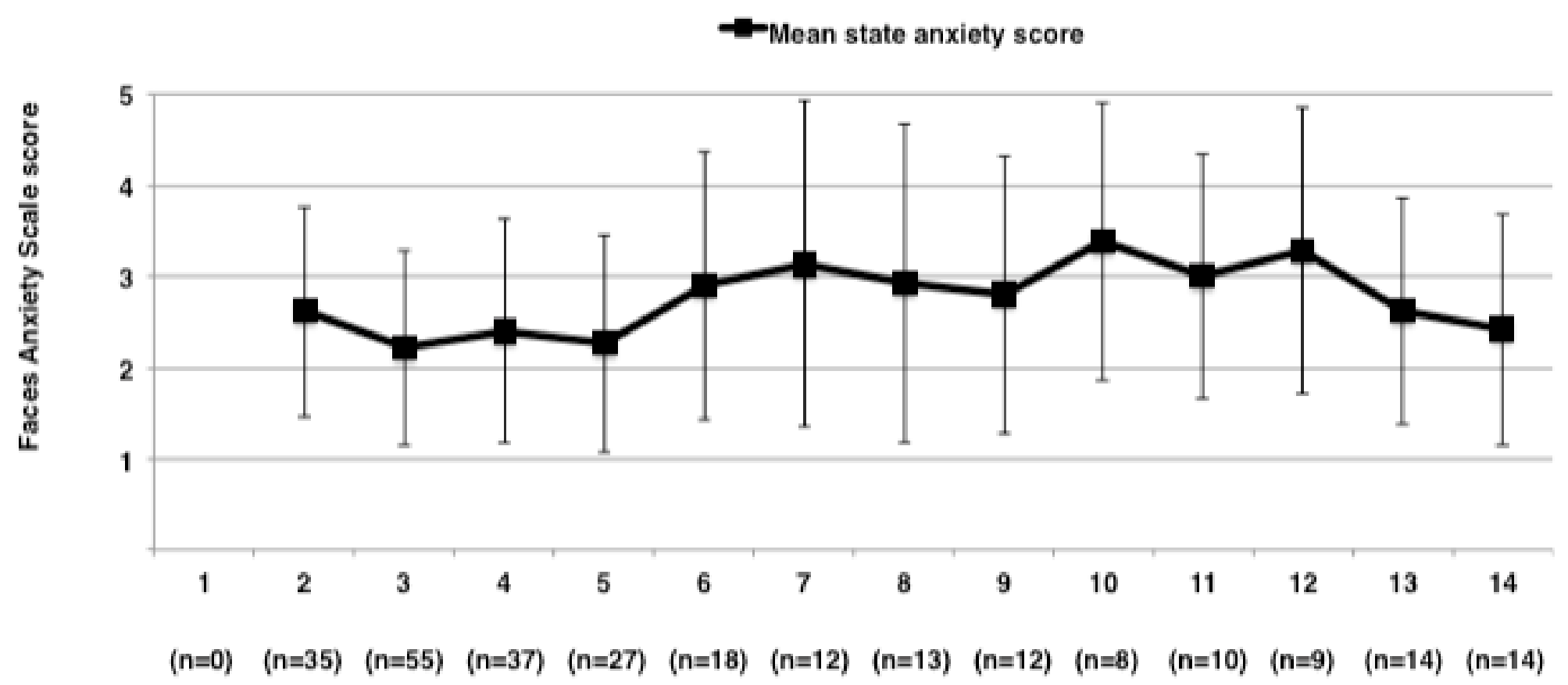

Days of stay in the intensive care unit and number of participants

Faces Anxiety Scale scores from 1 to 5 (1-2 = low anxiety, 3-5 = moderate to severe anxiety)

Figure 2. Mean state anxiety score and standard deviation for the days of stay in the intensive care unit 
Table 1. Multiple Linear Regression: Factors associated with state anxiety in intensive care patients (n=141)

\begin{tabular}{|c|c|c|c|c|c|c|}
\hline \multirow{2}{*}{ Variables } & \multicolumn{2}{|c|}{$\begin{array}{l}\text { Unstandardised } \\
\text { Coefficients }\end{array}$} & \multirow{2}{*}{$\begin{array}{c}\begin{array}{c}\text { Standardised } \\
\text { Coefficients }\end{array} \\
\text { Beta }\end{array}$} & \multirow{2}{*}{ Sig. } & \multicolumn{2}{|c|}{$\begin{array}{l}\text { 95.0\% Confidence } \\
\text { Interval for B }\end{array}$} \\
\hline & $B$ & Std. Error & & & $\begin{array}{l}\text { Lower } \\
\text { Bound }\end{array}$ & $\begin{array}{l}\text { Upper } \\
\text { Bound }\end{array}$ \\
\hline (Constant) & 1.204 & 0.317 & & 0.001 & 0.577 & 1.831 \\
\hline Trait anxiety & 0.027 & 0.008 & 0.294 & 0.001 & 0.011 & 0.043 \\
\hline Pain & 0.095 & 0.046 & 0.180 & 0.043 & 0.003 & 0.187 \\
\hline
\end{tabular}


Table 2. Univariate analysis: association between variables significant at $p \leq 0.2$ and trait anxiety score $(n=120)$

\begin{tabular}{ll}
\hline Variables/Trait anxiety score & $r$ value and $(p)$ \\
\hline Age & $-0.26(0.004)$ \\
Evidence of mental health treatment & $0.29(0.001)$ \\
Trait optimism & $-0.58(<0.001)$ \\
State anxiety & $0.32(<0.001)$ \\
Smoking & $0.30(0.001)$ \\
Length of hospital stay & $0.18(0.04)$ \\
Length of propofol infusion & $0.17(0.053)$ \\
Total dose of propofol & $0.15(0.09)$ \\
Total dose of midazolam & $0.18(0.052)$ \\
Total dose of morphine & $-0.16(0.08)$ \\
Length of fentanyl infusion & $0.19(0.04)$ \\
Total dose of oxycodone & $0.15(0.094)$ \\
Pain & $0.17(0.058)$ \\
\hline
\end{tabular}

Spearman's rank correlation coefficients, Mann-Whitney $U$ test, Kruskal-Wallis test were used with, respectively, continuous, binary and categorical risk factors 
Table 3. Multiple Linear Regression: Factors associated with trait anxiety in intensive care patients $(\mathbf{n}=120)$

\begin{tabular}{|c|c|c|c|c|c|c|}
\hline \multirow[b]{2}{*}{ Variables } & \multicolumn{2}{|c|}{ Unstandardised Coefficients } & \multirow{2}{*}{$\begin{array}{c}\text { Standardised Coefficients } \\
\text { Beta }\end{array}$} & \multirow[b]{2}{*}{ Sig. } & \multicolumn{2}{|c|}{ 95\% Confidence Interval for B } \\
\hline & B & Std. Error & & & Lower Bound & Upper Bound \\
\hline (Constant) & 62.369 & 4.209 & & 0.001 & 54.030 & 70.707 \\
\hline Trait optimism & -1.549 & 0.181 & -0.563 & 0.001 & -1.908 & -1.190 \\
\hline State anxiety & 2.235 & 0.728 & 0.204 & 0.003 & 0.792 & 3.678 \\
\hline Age & -0.142 & 0.049 & -0.188 & 0.004 & -0.238 & -0.045 \\
\hline $\begin{array}{l}\text { Evidence of mental } \\
\text { health treatment }\end{array}$ & 4.186 & 1.586 & 0.175 & 0.009 & 1.043 & 7.329 \\
\hline
\end{tabular}




\section{References}

American Psychiatric Association, 2013. Diagnostic and statistical manual of mental disorders. 5th ed. Washington, DC: Author.

Barr, J., Fraser, G.L., Puntillo, K., Ely, E.W., Gelinas, C., Dasta, J.F., Davidson, J.E., Devlin, J.W., Kress, J.P., Joffe, A.M., Coursin, D.B., Herr, D.L., Tung, A., Robinson, B.R., Fontaine, D.K., Ramsay, M.A., Riker, R.R., Sessler, C.N., Pun, B., Skrobik, Y., Jaeschke, R., 2013. Clinical practice guidelines for the management of pain, agitation, and delirium in adult patients in the intensive care unit. Crit Care Med 41 (1), 263-306.

Bryant, R.A., Creamer, M., O'Donnell, M., Silove, D., McFarlane, A.C., 2009. A study of the protective function of acute morphine administration on subsequent posttraumatic stress disorder. Biological Psychiatry 65 (5), 438-440.

Castillo, M.I., Aitken, L.M., Cooke, M.L., 2013. Study protocol: Intensive care anxiety and emotional recovery (Icare) — A prospective study. Australian Critical Care 26 (3), 142-147.

Castillo, M.I., Cooke, M.L., MacFarlane, B., Aitken, L.M., 2015a. In ICU state anxiety is not associated with posttraumatic stress symptoms over six months after ICU discharge: A prospective study. Australian Critical Care.

Castillo, M.I., Cooke, M.L., Macfarlane, B., Aitken, L.M., 2015b. Trait anxiety but not state anxiety during critical illness was associated with anxiety and depression over six months after ICU. Critical Care Medicine In press.

Chlan, L., 2003. Description of anxiety levels by individual differences and clinical factors in patients receiving mechanical ventilatory support. Heart \&amp; Lung: The Journal of Acute and Critical Care 32 (4), 275-282.

Chlan, L., Savik, K., 2011. Patterns of Anxiety in Critically Ill Patients Receiving Mechanical Ventilatory Support. Nursing Research 60, S50-57.

Chlan, L.L., 2004. Relationship between two anxiety instruments in patients receiving mechanical ventilatory support. Journal of Advanced Nursing 48 (5), 493-499.

Chlan, L.L., Weinert, C.R., Heiderscheit, A., Tracy, M.F., Skaar, D.J., Guttormson, J.L., Savik, K., 2013. Effects of patient-directed music intervention on anxiety and sedative exposure in critically ill patients receiving mechanical ventilatory support: a randomized clinical trial. JAMA 309 (22), 2335-2344.

Clark, D.M., Clark, D.M., Ehlers, A., McManus, F., Hackmann, A., 2003. Cognitive Therapy Versus Fluoxetine in Generalized Social Phobia: A Randomized Placebo-Controlled Trial. Journal of Consulting and Clinical Psychology 71 (6), 1058-1067.

Clewett, D., Bachman, S., Mather, M., 2014. Age-related reduced prefrontal-amygdala structural connectivity is associated with lower trait anxiety. Neuropsychology 28 (4), 631-642.

Cook, D.A., Joyce, C.J., Barnett, R.J., Birgan, S.P., Playford, H., Cockings, J.G., Hurford, R.W., 2002. Prospective independent validation of APACHE III models in an Australian tertiary adult intensive care unit. Anaesth Intensive Care 30 (3), 308-315.

Crawford, J., Cayley, C., Lovibond, P.F., Wilson, P.H., Hartley, C., 2011. Percentile Norms and Accompanying Interval Estimates from an Australian General Adult Population Sample for Self-Report Mood Scales (BAI, BDI, CRSD, CES-D, 
DASS, DASS-21, STAI-X, STAI-Y, SRDS, and SRAS). Australian Psychologist 46 (1), 3-14.

Doenges, M., Moorhouse, M., Murr, A., 2010. Nursing Diagnosis Manual: Planning, Individualizing, and Documenting Client Care. Philadelphia, PA, USA: F.A. Davis.

Ely, E.W., Margolin, R., Francis, J., May, L., Truman, B., Dittus, R., Speroff, T., Gautam, S., Bernard, G.R., Inouye, S.K., 2001. Evaluation of delirium in critically ill patients: validation of the Confusion Assessment Method for the Intensive Care Unit (CAM-ICU). Crit Care Med 29 (7), 1370-1379.

Faul, F., Erdfelder, E., Buchner, A., Lang, A.-G., 2009. Statistical power analyses using G*Power 3.1: Tests for correlation and regression analyses. Behavior Research Methods 41, 1149-1160.

Fletcher, S., Creamer, M., Forbes, D., 2010. Preventing post traumatic stress disorder: are drugs the answer? Australian and New Zealand Journal of Psychiatry 44 (12), 1064-1071.

Gelinas, C., Fillion, L., Puntillo, K.A., Viens, C., Fortier, M., 2006. Validation of the critical-care pain observation tool in adult patients. Am J Crit Care 15 (4), 420427.

Gelinas, C., Harel, F., Fillion, L., Puntillo, K.A., Johnston, C.C., 2009. Sensitivity and specificity of the critical-care pain observation tool for the detection of pain in intubated adults after cardiac surgery. J Pain Symptom Manage 37 (1), 58-67.

IBMCorp, 2013. IBM SPSS Statistics for Windows. IBM Corp, Armonk, NY.

Jackson, J.J., Hill, P.L., Payne, B.R., Roberts, B.W., Stine-Morrow, E.A., 2012. Can an old dog learn (and want to experience) new tricks? Cognitive training increases openness to experience in older adults. Psychology and Aging 27 (2), 286-292.

Jones, C., Skirrow, P., Griffiths, R.D., Humphris, G.H., Ingleby, S., Eddleston, J., Waldmann, C., Gager, M., 2003. Rehabilitation after critical illness: a randomized, controlled trial. Critical Care Medicine 31 (10), 2456-2461.

Kepka, S., Baumann, C., Anota, A., Buron, G., Spitz, E., Auquier, P., Guillemin, F., Mercier, M., 2013. The relationship between traits optimism and anxiety and health-related quality of life in patients hospitalized for chronic diseases: data from the SATISQOL study. Health Qual Life Outcomes 11, 134.

Kress, J.P., Gehlbach, B., Lacy, M., Pliskin, N., Pohlman, A.S., Hall, J.B., 2003. The long-term psychological effects of daily sedative interruption on critically ill patients. American Journal of Respiratory and Critical Care Medicine 168 (12), $1457-1461$.

McKinley, S., Madronio, C., 2008. Validity of the Faces Anxiety Scale for the assessment of state anxiety in intensive care patients not receiving mechanical ventilation. Journal Of Psychosomatic Research 64 (5), 503-507.

McKinley, S., Stein-Parbury, J., Chehelnabi, A., Lovas, J., 2004. Assessment of anxiety in intensive care patients by using the Faces Anxiety Scale. American Journal of Critical Care 13 (2), 146-152.

Moser, D.K., Chung, M.L., McKinley, S., Riegel, B., An, K., Cherrington, C.C., Blakely, W., Biddle, M., Frazier, S.K., Garvin, B.J., 2003. Critical care nursing practice regarding patient anxiety assessment and management. Intensive \& Critical Care Nursing 19 (5), 276-288.

Moser, D.K., Dracup, K., 1996. Is anxiety early after myocardial infarction associated with subsequent ischemic and arrhythmic events? Psychosomatic Medicine 58 (5), 395-401. 
Myhren, H., Ekeberg, O., Tøien, K., Karlsson, S., Stokland, O., 2010. Posttraumatic stress, anxiety and depression symptoms in patients during the first year post intensive care unit discharge. Critical Care [NLM - MEDLINE] 14 (1), 0.

Myhren, H., Tøien, K., Ekeberg, O., Karlsson, S., Sandvik, L., Stokland, O., 2009. Patients' memory and psychological distress after ICU stay compared with expectations of the relatives. Intensive Care Medicine 35 (12), 2078-2086.

Nelson, B.J., Weinert, C.R., Bury, C.L., Marinelli, W.A., Gross, C.R., 2000. Intensive care unit drug use and subsequent quality of life in acute lung injury patients. Critical Care Medicine 28 (11), 3626-3630.

O'Brien, J.L., Moser, D.K., Riegel, B., Frazier, S.K., Garvin, B.J., Kim, K.A., 2001. Comparison of anxiety assessments between clinicians and patients with acute myocardial infarction in cardiac critical care units. American Journal Of Critical Care: An Official Publication, American Association Of Critical-Care Nurses 10 (2), 97-103.

Peris, A., Bonizzoli, M., Iozzelli, D., Migliaccio, M.L., Zagli, G., Bacchereti, A., Debolini, M., Vannini, E., Solaro, M., Balzi, I., Bendoni, E., Bacchi, I., Trevisan, M., Giovannini, V., Belloni, L., 2011. Early intra-intensive care unit psychological intervention promotes recovery from post traumatic stress disorders, anxiety and depression symptoms in critically ill patients. Critical Care (London, England) 15 (1), R41-R41.

Puntillo, K.A., Arai, S., Cohen, N.H., Gropper, M.A., Neuhaus, J., Paul, S.M., Miaskowski, C., 2010. Symptoms experienced by intensive care unit patients at high risk of dying. Crit Care Med 38 (11), 2155-2160.

Raikkonen, K., Matthews, K.A., Flory, J.D., Owens, J.F., Gump, B.B., 1999. Effects of optimism, pessimism, and trait anxiety on ambulatory blood pressure and mood during everyday life. J Pers Soc Psychol 76 (1), 104-113.

Ristvedt, S.L., Trinkaus, K.M., 2009. Trait anxiety as an independent predictor of poor health-related quality of life and post-traumatic stress symptoms in rectal cancer. Br J Health Psychol 14 (Pt 4), 701-715.

Rotondi, A.J., Chelluri, L., Sirio, C., Mendelsohn, A., Schulz, R., Belle, S., Im, K., Donahoe, M., Pinsky, M.R., 2002. Patients' recollections of stressful experiences while receiving prolonged mechanical ventilation in an intensive care unit. Critical Care Medicine 30 (4), 746-752.

Scheier, M.F., Carver, C.S., Bridges, M.W., 1994. Distinguishing optimism from neuroticism (and trait anxiety, self-mastery, and self-esteem): a reevaluation of the Life Orientation Test. J Pers Soc Psychol 67 (6), 1063-1078.

Schelling, G., Briegel, J., Roozendaal, B., Stoll, C., Rothenhäusler, H.B., Kapfhammer, H.P., 2001. The effect of stress doses of hydrocortisone during septic shock on posttraumatic stress disorder in survivors. Biological Psychiatry 50 (12), 978985.

Spielberger, C.D., 1983. Manual for the State-Trait Anxiety Inventory (Form Y). Mind Garden, Palo Alto, CA.

Spielberger, C.D., 1966. Theory and research on anxiety. In: Spielberger, C.D. (Ed.), Anxiety and behavior. Academic Press, New York, pp. 3-19.

Spielberger, C.D., Reheiser, E.C., 2009. Assessment of Emotions: Anxiety, Anger, Depression, and Curiosity. Applied Psychology: Health and Well-Being 1 (3), 271-302.

StataCorp, 2013. Stata Statistical Software: Release 13. StataCorp LP, College Station, TX. 
Steimer, T., 2002. The biology of fear- and anxiety-related behaviors. Dialogues Clin Neurosci 4 (3), 231-249.

Tang, T.Z., DeRubeis, R.J., Hollon, S.D., Amsterdam, J., Shelton, R., Schalet, B., 2009. Personality change during depression treatment: a placebo-controlled trial. Archives of General Psychiatry 66 (12), 1322-1330.

Tate, J.A., Devito Dabbs, A., Hoffman, L.A., Milbrandt, E., Happ, M.B., 2011. Anxiety and Agitation in Mechanically Ventilated Patients. Qualitative Health Research.

Tate, J.A., Devito Dabbs, A., Hoffman, L.A., Milbrandt, E., Happ, M.B., 2012. Anxiety and agitation in mechanically ventilated patients. Qualitative Health Research 22 (2), 157-173.

Yava, A., Tosun, N., Ünver, V., Çiçek, H., 2011. Patient and nurse perceptions of stressors in the intensive care unit. Stress and Health 27 (2), e36-e47. 